\title{
Terapia de Exposición Mediante Realidad Virtual e Internet en el Trastorno de Ansiedad/Fobia Social: Una Revisión Cualitativa
}

\author{
Virtual Reality Exposure Therapy and Internet in Social Anxiety Disorder: A Review.
}

\author{
Emilio S. García-García \\ Ana I. Rosa-Alcázar \\ $\&$ \\ Pablo J. Olivares-Olivares \\ Universidad de Murcia, España
}

(Rec: 10 de Agosto de 2011 / Acep: 12 de Octubre de 2011)

\begin{abstract}
Resumen
La Terapia de Exposición mediante Realidad Virtual e Internet constituyen dos nuevos formatos de aplicación de la terapia de conducta y constituyen una alternativa a la exposición en vivo estándar. El presente trabajo estudia la relevancia de estas modalidades de tratamiento en el Trastorno por Ansiedad/Fobia Social. Se ha llevado a cabo una búsqueda de estudios realizados al respecto, concluyéndose tanto que la evidencia disponible muestra indicios razonables de su efectividad como que resultan necesarios nuevos estudios con mayores tamaños muestrales.
\end{abstract}

Palabras clave: Realidad virtual, terapia de exposición en realidad virtual, internet,

fobia social, ansiedad social.

\begin{abstract}
Virtual reality exposure therapy and the Internet are two new formats for the application of behavior therapy and are an alternative to standard in vivo exposure. The present study examines the relevance of these treatment modalities in Social Anxiety/Phobia Disorder. It has carried out a search of the studies performed, thus concluding that the evidence shows prima facie evidence of its effectiveness as a resulting need for new studies with larger sample sizes.
\end{abstract}

Key words: Virtual reality, virtual reality exposure therapy, internet, social phobia, social anxiety. 


\section{Introducción}

El Trastorno por Ansiedad/Fobia Social (TAS, en adelante) se integra en el espectro de los trastornos de ansiedad, centrándose en el miedo a la evaluación negativa que puede experimentar una persona en distintas situaciones de relación/actuación social (Olivares, 2009). Estas situaciones hacen que la persona se sienta insegura, experimentando sensaciones muy intensas y desagradables que terminan interfiriendo de modo significativo en su actividad cotidiana (American Psychiatric Association, 2000).

Tomando en consideración los datos disponibles más recientes, se estima que entre el $7 \%$ y el $13 \%$ de la población en las sociedades occidentales cumplen los criterios requeridos para el diagnóstico de TAS en algún momento de sus vidas (véase Bados, 2009; Furmark et al., 2002; Kessler, Berglund, Demler, Jin \& Walters, 2005; Rao, Beidel, Turner, Ammerman, Crosby \& Sallee, 2007), lo que hace del TAS el tercer trastorno psicológico más frecuente en las sociedades occidentales, tras la depresión y el consumo de alcohol (Amerigen, Mancini \& Farvolden, 2003; Ruscio, Brown, Chiu, Sareen, Stein \& Kessler, 2008), así como un importante problema de salud mental (Wittchen, Feutsch, Sonntag, Muller \& Liebowitz, 2000) y de salud pública (Olivares, 2005) que afecta de manera muy significativa a la merma de la calidad de vida (Eng, Coles, Heimberg \& Safren, 2001; Hambrick, Turk, Heimberg, Schneier \& Liebowitz, 2003; Kessler, 2003; Safren, Heimberg, Brown \& Holle, 1997; Wittchen et al., 2000) y conlleva importantes costes económicos para los sistemas de salud pública que Greenberg et al. (1999), en los EE.UU de Norteamérica, cuantificaron en más de 42 millones de dólares/año. Además, toda esta problemática se ve considerablemente agravada tanto por la elevada comorbilidad del trastorno como por su cronicidad. En relación con la comorbilidad, Kessler, Chiu, Demler \& Walters (2005) informaron que los trastornos de ansiedad se asocian con altas tasas de comorbilidad con otros trastornos de ansiedad y depresión que, en su estudio, se sitúan entre el $40 \%$ y el $80 \%$. Respecto de la cronicidad, los datos disponibles permiten sostener que ésta es en general moderadamente estable a lo largo de la vida (así Beidel \& Turner, 2005; Pine, Cohen, Gurley, Brook \& Ma, 1998; Rapee \& Spence, 2004; Rucio, Brown, Chiu, Sareen, Stein \& Kessler, 2008).

Pese a que puede tener un inicio temprano (véase por ejemplo Rosa-Alcázar, Olivares \& Olivares-Olivares, 2007), éste se sitúa generalmente en la adolescencia. La intensa ansiedad en situaciones de relación/actuación social, resulta especialmente perturbadora en los jóvenes, para quienes es de capital importancia la pertenencia a un grupo o el inicio de relaciones laborales y de pareja; esta interferencia puede terminar generando un consumo desproporcionado de sustancias tóxicas de curso legal e ilegal (Katon \& Geyman, 2007 citados en Rakel, 2007). La preocupación de los investigadores y clínicos por este trastorno tiene argumentos de peso (Beidel \& Turner, 2005; Olivares-Olivares, RosaAlcázar y Olivares, 2007), pues a la elevada prevalencia del trastorno se añaden las repercusiones que éste tiene también para la salud física (mayor probabilidad de inicio y abuso del consumo de sustancias tóxicas y alcohol), la ocurrencia de trastornos psicopatológicos comórbidos (depresión, suicidio, trastornos de ansiedad), en el desarrollo social (problemas de inserción y mantenimiento en el mundo laboral, dependencia económica de las familias o problemas para iniciar y mantener relaciones de pareja) y en el rendimiento intelectual (disminución del rendimiento escolar o académico y abandono de los estudios). Por todo ello, el TAS es reconocido hoy en día como una condición crónica e incapacitante, enfatizándose la necesidad de su detección y tratamiento temprano (Olivares, Rosa \& Piqueras, 2005).

Las revisiones meta-analíticas son, en general, consistentes en mostrar que los Tratamientos CognitivoConductuales (TCC, en adelante) aportan importantes cambios en las personas con TAS. Así, Taylor (1996) encontró que la reestructuración cognitiva (RC, en adelante), el entrenamiento en habilidades sociales (HHSS, en adelante), la exposición (Exp, en adelante) y la Exp combinada con $\mathrm{RC}$, resultaron superiores a la lista de espera en las medidas de autorregistro de las respuestas alteradas asociadas a este trastorno. Además, la valoración del efecto de estos tratamientos aumentó significativamente tras un periodo de tres meses (post-tratamiento). No hubo diferencias significativas entre los efectos de los diferentes tipos de TCC aplicados.

Federoff \& Taylor (2001), compararon el seguimiento de TCC en TAS: Exp, RC, Exp con RC, entrenamiento en HHSS y relajación. Aunque todos los tratamientos fueron moderadamente efectivos en el post-tratamiento y mostraron efectos significativos en el seguimiento, no hubo diferencias significativas entre las diferentes intervenciones. De un interés particular es la relativa importancia de la RC y Exp para superar los temores y el funcionamiento social. En cuanto a la pregunta de si es la Exp suficiente para producir buenos resultados clínicos o si es necesaria la RC para maximizar los beneficios del tratamiento, Feske \& Chambless (1995) dirigieron esta pregunta a una revisión meta-analítica para comparar tratamientos que usaron sólo Exp, con tratamientos que combinaron Exp con RC. En base a 21 estudios, la Exp sola y la Exp combinada con RC produjeron un efecto equivalente en el pre, post-tratamiento y seguimiento en auto-informes de resultados; además, no se hallaron diferencias en cuanto a abandonos entre los dos tipos de tratamientos. En otro meta-análisis, Gould, Buckminster, Pollack, Otto \& Yap (1997) revisaron 16 estudios que usaron TCC encontrándose que, entre las variaciones de los TCC examinados, los que tenían un componente de Exp administrado como único recurso o en combinación con RC presentaban los mayores tamaños del efecto; los que usaron sólo RC o sólo entrenamiento en HHSS fueron 
Terapia de Exposición Mediante Realidad Virtual e Internet en el Trastorno de Ansiedad/Fobia Social:

UnA REvisión CuALITATIVA

Tabla 1: Resumen de estudios incluidos en la Revisión

\begin{tabular}{|c|c|c|c|c|c|}
\hline Estudio & Autores/Año & $\mathrm{N}$ & Sesiones & Instrumentos de Evaluación & Grupo Control \\
\hline & North, M., North, S. y Coble, J., 1998 & 16 & 5 & ATPS, SUDS & Placebo \\
\hline & Harris, S., Kemmerling, R. y North, M., 2002 & 14 & 4 & LSAS, STAI, PRCS, ATPS, SUDS & Lista de Espera \\
\hline & Pertaub, D.P, Slater, M. y Barker, C., 2002 & 40 & 6 & MPRCS, PRCS, SADS, FNE, SCL-90-R & --- \\
\hline 4 & $\begin{array}{l}\text { Roy, S., Klinger, E., Légeron, P., Lauer, F., Chemin, } \\
\text { I. y Nugues, P., } 2003\end{array}$ & 10 & 12 & $\begin{array}{l}\text { LSAS, HAD, BDI-13, RAS, SCIA, SIS, } \\
\text { SISST, CGI }\end{array}$ & Lista de Espera \\
\hline 5 & $\begin{array}{l}\text { James, L., Lin, C., Steed, A., Swapp, D. y Slater, M., } \\
2003\end{array}$ & 10 & 2 & SADS & --- \\
\hline 6 & $\begin{array}{l}\text { Garau, M., Slater, M., Vinayagamoorthy, V., Brogni, } \\
\text { A., Steed, A. y Sasse, M., } 2003\end{array}$ & 48 & 1 & SADS & --- \\
\hline 7 & Slater, M., Pertaub, D.P., Barker, C. y Clark, D., 2004 & 36 & 2 & PRCS & Sin FS \\
\hline 8 & $\begin{array}{l}\text { Klinger, E., Bouchard, S., Légeron, P., Roy, S., Lauer, } \\
\text { F., Chemin, I. y Nugues, P., } 2005\end{array}$ & 36 & 12 & $\begin{array}{l}\text { LSAS, HAD, BDI-13, RAS, SISST, SIS, } \\
\text { CGI, SCIA }\end{array}$ & TCC \\
\hline 9 & $\begin{array}{l}\text { Anderson, P.L., Zimand, E., Hodges, L.F. y Rothbaum, } \\
\text { B.O., } 2005\end{array}$ & 10 & 8 & PRCS, PRCA, SSPS-P, SSPS-N & --- \\
\hline 10 & Herbelin, B., 2005 & 200 & 1 & LSAS, SADS, FQ-S & Media FS \\
\hline 11 & $\begin{array}{l}\text { Garau, M., Slater, M., Pertaub, D.P. y Razzaque, S., } \\
2005\end{array}$ & 41 & 4 & POMS, SAD & --- \\
\hline 12 & Slater, M. , et al., 2006 & 20 & 1 (3 fases) & SPQ & Baja FS \\
\hline 13 & $\begin{array}{l}\text { Grillon, H., Riquier, F., Herbelin, B. y Thalmann, } \\
\text { D., } 2006\end{array}$ & 8 & 8 & FQ, LSAS, SISST, BDI & --- \\
\hline 14 & Pan, X. y Slater, M., 2007 & 24 & 1 (3 fases) & SPAI & --- \\
\hline 15 & $\begin{array}{l}\text { Anderson, P., Zimand, E., Schmertz, S.K. y Ferrer, } \\
\text { M., } 2007\end{array}$ & 10 & 8 & $\begin{array}{l}\text { FNE, PRCA, PRCS, SSPS-P, SSPS-N, } \\
\text { BDI, STAI-S, STAI-T. }\end{array}$ & --- \\
\hline 16 & $\begin{array}{l}\text { Botella, C., Guillén, V., García-Palacios, A., Gallego, } \\
\text { M.J., Baños, R.M. y Alcañiz, M., } 2007\end{array}$ & 12 & 7,58 & ADIS-IV-L, FPSQ, PSSEQ, SUDS & --- \\
\hline 17 & $\begin{array}{l}\text { Carlbring, P., Gunnardsdóttir, M., Hedensjö, L., } \\
\text { Andersson, G., Ekselius, L. y Furmark, T., } 2007\end{array}$ & 57 & 9 & $\begin{array}{l}\text { LSAS, SPS, SIAS, SPSQ, BAI, } \\
\text { MADRS, SCID. }\end{array}$ & Lista de Espera \\
\hline 18 & $\begin{array}{l}\text { Tillfords, M., Carlbring, P., Furmark, T., Lewenhaupt, } \\
\text { S., Spak, M., Erikson A., Westling, B.E. y Andersson, } \\
\text { G., } 2008\end{array}$ & 65 & 9 & LSAS, SPS, SIAS, SPSQ. & --- \\
\hline 19 & Kang, S., Gratch, J., Wang, N. y Watt, J.H., 2008 & 131 & 4 & SRS, SS, ES, TLS. & --- \\
\hline 20 & Mühlberger, A., Wiesser, M.J. y Pauli, P., 2008 & 26 & 2 & SPAI, STAI-T, STAI-S, PANAS. & Lista de Espera \\
\hline 21 & Berger, T., Holh, E. y Caspar, F., 2009 & 52 & 10 & LSAS, SPS, SIAS, SCL-90-R, BDI. & Lista de Espera \\
\hline 22 & Brinkman, W.P., Inan, F. y Van Der Mast, C., 2009 & 22 & 1 & LSAS, IPQ. & --- \\
\hline 23 & Wallach, H.S., Safir, M.P. y Bar-Zvi, M., 2010 & 88 & 12 & LSAS, SSPS-N, SSPS-P, FNE & TCC \\
\hline 24 & $\begin{array}{l}\text { Dumoulin, S., Bouchard, S., Robillard, G., Guitard, } \\
\text { T. y Klinger, E., } 2010\end{array}$ & 45 & 14 & $\begin{array}{l}\text { LSAS, RAS, SPS, ASC, FNE, BDI-II, } \\
\text { STAI }\end{array}$ & $\begin{array}{c}\text { TCC } \\
\text { Lista de Espera }\end{array}$ \\
\hline
\end{tabular}

Estudios relacionados con el miedo a hablar en público Estudios relacionados con entornos públicos virtuales Estudios relacionados con entrevistas de trabajo

Estudios relacionados con Internet y Ordenadores (PC) 
menos eficaces, produciendo tamaños del efecto medios o bajos. Estos hallazgos aportan evidencia empírica sobre la importancia de la Exp en el tratamiento del TAS.

Por tanto, la posibilidad de trabajar con la Exp de forma controlada así como la necesidad de diseñar tratamientos que incrementen su eficacia, ha llevado a clínicos e investigadores a considerar como herramienta prometedora el uso de dispositivos electrónicos y toda la tecnología de que se dispone actualmente. Es así como la Realidad Virtual (RV, en adelante) e Internet ofrecen un nuevo paradigma de interacción en donde los usuarios no son sólo observadores de imágenes sino que son participantes activos de un mundo virtual tridimensional. Este sistema ha sido propuesto como un efectivo método de Exp y desde hace bastante tiempo ha mostrado indicios satisfactorios sobre su eficacia para el tratamiento de algunas fobias simples como la acrofobia y la fobia a volar (Olasov \& Hodges, 1999) y sus posibilidades en relación con el tratamiento del miedo más frecuente de entre los que integran la fobia/ansiedad social: el miedo a hablar en público (Pertaub, Slater \& Barker, 2001); estos autores hallaron que el miedo a hablar en público también podía ser inducido usando RV y que éste guardaba una relación directa con el tipo de feedback que daba la audiencia al participante.

En las últimas décadas se ha producido un aumento de los estudios centrados en el TAS, empleando diversas técnicas de RV e Internet. Así, se han desarrollado entornos virtuales que provocan ansiedad para el tratamiento de pacientes con miedo a hablar en público (Botella, Guillén, García-Palacios, Gallego, Baños \& Alcañiz, 2007; Lee et al., 2002), pero al día de hoy se desconoce cuáles son las intervenciones más eficaces en el tratamiento del TAS y cuáles son sus características más relevantes en relación con su eficacia. Aunque se han llevado a cabo revisiones y meta-análisis del empleo de la RV en diversos desórdenes de ansiedad (Krijn, Emmelkamp, Olafsson \& Biemond, 2004; Parsons \& Rizzo, 2008), todavía no se ha realizado ninguna referida exclusivamente al TAS. En consonancia con esta carencia, el presente artículo pretende ser una respuesta a esta falta de información y el primer trabajo de revisión en español sobre el empleo de la RV e Internet en el tratamiento del TAS.

\section{Método}

Se realizó una revisión de la literatura entre 19952010, ya que es precisamente a mediados de los años noventa cuando empiezan a aparecer sistemas y equipos informáticos que permiten la recreación de escenarios virtuales de calidad. Se realizó una búsqueda en las bases de datos Academic Search Premier, EBSCO host, ISI Web of Knowledge, MEDLINE, PubMed, PSICODOC y Google Académico, utilizando como palabras clave -key words«virtual reality», «virtual reality therapy» 0 «virtual reality exposure therapy», combinadas con «Internet» $\mathrm{y}$ «social anxiety» $\mathrm{O}$ «social phobia». Además se revisó la bibliografía de los artículos a los que se tuvo acceso a texto completo para localizar nuevas investigaciones. Los criterios de inclusión de los trabajos fueron:

- Al menos dos momentos de medida (pre y postratamiento).

- Población de interés: jóvenes, mayores de edad, tanto hombres como mujeres y que tuviesen presente TAS, descartando aquellos sujetos con otros trastornos o presentasen alteraciones del comportamiento significativas.

- Idioma: inglés, español o francés.

En total se seleccionaron 24 estudios, que aparecen resumidos en la tabla 1. En ella, se presentan ordenados cronológicamente y considerando:

1. Identificación del trabajo.

2. Autores y año de publicación.

3. Número de participantes.

4. Número de sesiones.

5. Instrumentos de evaluación.

6. Grupo Control.

\section{Resultados}

\section{Análisis descriptivo de los estudios}

Se han considerado las investigaciones que han aplicado la RV y/o Internet para el tratamiento del TAS. Entre éstos se hallan:

- Miedo a hablar en Público: Los experimentos estudiados, recrean un entorno virtual parecido a un aula o auditorio, donde puede llevarse a cabo una conferencia delante de un grupo de personas virtuales (avatares). Dichos avatares pueden presentar diferentes tipos de comportamientos y actitudes, intentando elicitar ansiedad en la persona que debe hablar ante ellos.

En el estudio de North, North \& Coble (1998) -estudio 1 según tabla 1-, las experiencias en RV se llevaron a cabo en el Laboratorio de RV de la Universidad Clark de Atlanta (EE.UU.). La escena virtual consistía en la simulación de un auditorio situado en el Edificio de Ciencias de la Investigación de la propia Universidad. El área de asientos del auditorio podía albergar hasta 100 avatares. El programa de ordenador para RV generaba una jerarquía de situaciones provocadoras de temor para hablar ante el público, que el participante captaba a través del HMD (Head-Mounted Display), dispositivo colocado en la cabeza donde podían verse imágenes tridimensionales de la audiencia según los movimientos que se realizaban. El grupo experimental estaba formado por 6 sujetos $(N=6)$ y el grupo control por 8 . Se produjeron 2 abandonos. Los instrumentos de medida empleados fueron el ATPS y SUDS (ver tabla 2). Se realizaron 5 sesiones semanales (con una media de 15 minutos cada sesión) de tratamiento. 
Tabla 2: Resumen de instrumentos de evaluación empleados en los estudios

\begin{tabular}{|c|c|c|}
\hline Siglas & Instrumento de Evaluación & Estudios donde se emplea \\
\hline ADIS-IV-L & Anxiety Disorders Interview Schedule Lifetime & 16 \\
\hline ASC & Altered States of Consciousness & 24 \\
\hline ATPS & Attitude Towards Public Speaking & 1,2 \\
\hline BAI & Beck Anxiety lnventory & 17 \\
\hline BDI & Beck Depression Inventory & $13,15,21$ \\
\hline BDI-II & Beck Depression Inventory - Second Edition & 24 \\
\hline BDI-13 & Beck Depression Inventory - 13 & 4,8 \\
\hline CGI & Escala de Impresión Clínica Global & 4,8 \\
\hline ES & Embarrassment Scale & 19 \\
\hline HAD & Hospital Anxiety-Depression & 4,8 \\
\hline IPQ & Illness Perception Questionnaire & 22 \\
\hline FQ & Fear Questionnarie & 13 \\
\hline FQ-S & Fear Questionnarie-Social & 10 \\
\hline FNE & Fear of Negative Evaluation & $3,15,23,24$ \\
\hline FPSQ & Fear of Public Speaking Questionnaire & 16 \\
\hline LSAS & Liebowitz Social Anxiety Scale & $2,4,8,10,13,17,18,21,22,23,24$ \\
\hline MADRS & Montgomery Åsberg Depression Rating Scale & 17 \\
\hline MPRCS & Modified Personal Report of Confidence as Speaker & 3 \\
\hline PANAS & Positive and Negative Affect Schedule & 20 \\
\hline POMS & Cuestionario «Perfil de los Estados de Ánimo» & 11 \\
\hline PSSEQ & Public Speaking Self-Efficacy Questionnaire & 16 \\
\hline PRCA & Personal Report of Communication Apprehension & 9,15 \\
\hline PRCS & Personal Report of Confidence as Speaker & $2,3,7,9,15$ \\
\hline RAS & Rathus Assertivennes Schedule & $4,8,24$ \\
\hline SADS & Standard Socially Affective Disorder Scale & $3,5,6,10,11$ \\
\hline SCIA & Social Contexts Inducing Anxiety & 4,8 \\
\hline SCID & Entrevista clínica estructurada para los trastornos de la personalidad & 17 \\
\hline SCL-90-R & Sympton Checklist-90-revised & 3 \\
\hline SIAS & Social Interaction Anxiety Scale & $17,18,21$ \\
\hline SIS & Sheehan Incapacity Scale & 4,8 \\
\hline SISST & Social Interaction Self-Statement Test & $4,8,13$ \\
\hline SPAI & Social Phobia Anxiety Inventory & 14,20 \\
\hline SPQ & Social Phobia Questionnaire & 12 \\
\hline SPS & Social Phobia Scale & $17,18,21,24$ \\
\hline SPSQ & Social Phobia Screening Questionnaire & 17 \\
\hline SRS & Self-Reported Rapport Scale & 19 \\
\hline SS & Self-Perfomance Scale & 19 \\
\hline SSPS-N & Self-Statements During Public Speaking-Negative & $9,15,23$ \\
\hline SSPS-P & Self-Statements During Public Speaking-Positive & $9,15,23$ \\
\hline STAI & State-Trait Anxiety Inventory & 2,24 \\
\hline STAI-S & STAI-State & 15,20 \\
\hline STAI-T & STAI-Trait & 15,20 \\
\hline SUDS & Subjetive Units of Disturbance Scale & $1,2,3,16$ \\
\hline TLS & Trustworthiness and Likableness Scale & 19 \\
\hline
\end{tabular}


En el estudio $n^{\circ} 2$ según tabla 1 (Harris, Kemmerling $\&$ North, 2002), se contó con un total de 14 sujetos, de los cuales 8 pertenecían al grupo experimental $(N=8)$ y 6 al grupo control (lista de espera). Los participantes del grupo experimental se sometieron a cuatro sesiones de tratamiento individual en RV, usando la escena de un auditorio virtual que veían a través del $H M D$. El terapeuta manipulaba el escenario virtual en cada una de las sesiones, modificando la conducta de los avatares para provocar estímulos ansiosos en el participante. Los instrumentos de medida empleados fueron el PRCS, STAI, LSAS, ATPS y SUDS (ver tabla 2). El tratamiento tuvo una duración de 4 sesiones (con una media de 12-15 minutos cada sesión).

Para el estudio de Pertaub, Slater \& Barker (2002) -estudio 3 de tabla $1-$, se plantearon 3 grupos experimentales $(n=40)$, de los cuales el grupo 1 debía hablar ante una audiencia con características de atención "neutral" y luego ante una audiencia "estática" $(n=12)$. El grupo 2, primero debía dirigirse a una audiencia con actitud "amigable" y luego a una audiencia con rasgos "hostiles" $(n=14)$. Finalmente, el grupo 3 debía actuar primero ante una audiencia "hostil" y luego ante una "amigable" $(n=14)$. Para este estudio los instrumentos de medida empleados fueron el FNE, MPRCS, PRCS, SADS y SCL-90-R (ver tabla 2). Se realizaron 2 sesiones por grupo y la audiencia virtual consistió en un grupo formado por 8 avatares masculinos formalmente vestidos sentados en una mesa haciendo un semicírculo. Los participantes de los tres grupos anteriormente citados, ofrecieron dos conversaciones a esta audiencia virtual, que podía presentar rasgos de actitud neutral, hostil o amigable. A todos los sujetos se les colocó un $H M D$ con sonido estereofónico para provocar la inmersión dentro de las escenas virtuales.

En el estudio de Slater, Pertaub, Barker \& Clark (2004) -estudio 7 de tabla 1-, se empleó como instrumento de medida el PRCS. El tamaño muestral $(\mathrm{N}=36)$ se encontraba dividido en dos grupos: uno de sujetos con TAS $(N=16)$ y otro grupo de no fóbicos sociales $(\mathrm{N}=20)$. El experimento consistía en hablar en dos escenarios virtuales. El primero estaba formado por una habitación con una mesa y varias sillas (que presentaban el aspecto de un pequeño seminario o reunión) y el segundo era la misma sala-seminario sólo que ahora era ocupada por 5 avatares (dos mujeres y tres hombres) sentados alrededor de la mesa. Su actitud era neutral ante el hablante (en este caso el sujeto experimental).

En el estudio ${ }^{\circ} 9$ de Anderson, Zimand, Hodges \& Rothbaum (2005) -ver tabla 1-, el grupo experimental estaba formado por 10 sujetos $(N=10)$. Las cuatro sesiones de $\mathrm{RV}$ consistían en dar una charla a un auditorio formado por 22 personas filmadas en un vídeo digital de alta resolución. El participante disponía de un $H M D$ para la inmersión en el mundo virtual mientras el terapeuta podía controlar las reacciones de la audiencia (interés, aburrimiento, aplausos, etc.). Los instrumentos de medida fueron el PRCA, PRCS,
SSPS-P y SSPS-N (ver tabla 2). 8 sesiones constituían el tratamiento ( 4 de entrenamiento en el manejo de la ansiedad y 4 de exposición empleando RV).

El estudio 10 reflejado en la tabla 1 (Herbelin, 2005), contó con una muestra de 200 sujetos de los cuales 100 constituyeron un grupo que no presentaba ansiedad social $(N=100), 58$ formaron el grupo con ansiedad social media $(N=58)$ y 42 el grupo con marcada fobia social $(N=42)$. Para su distribución en los tres grupos se emplearon el FQS, LSAS y $S A D S$ como instrumentos de medida. La única sesión de tratamiento tuvo una duración de exposición virtual de 1'45" aproximadamente y consistía en exponerse ante un "tribunal evaluador virtual" formado por 3 avatares masculinos y 2 femeninos, con diferentes actitudes (fastidio, mirada inquisidora, etc.)

- Entornos públicos virtuales: Integran escenarios donde el sujeto tiene alta probabilidad de relacionarse con personas desconocidas. Puede ser dentro del metro, un bar, una cafetería, etc.

El estudio $n^{\circ} 4$ de la tabla 1 (Roy, Klinger, Légeron, Lauer, Chemin \& Nugues, 2003), incluía cuatro situaciones de exposición virtual para evaluar cuatro aspectos fundamentales de la interacción: asertividad, interacción, intimidad y examen por parte de los otros, donde los pacientes $(N=4)$ se sometían a 12 sesiones de terapia virtual con una duración inferior a los 20 minutos de exposición. Cada sesión era individual y dirigida por un psicoterapeuta. Los instrumentos de evaluación empleados fueron el $B D I-13$, CGI, HAD, LSAS, SCIA, SIS, SISST y RAS (ver tabla 2). El grupo control era de lista de espera con $\mathrm{N}=6$.

En el estudio 5 (James, Lin, Steed, Swapp \& Slater, 2003) -ver tabla $1-$, los sujetos $(N=10)$ fueron expuestos a dos escenarios virtuales: uno de ellos una simulación de un vagón del metro de Londres y el otro un bar de copas. Se intentaba valorar el nivel de participación del sujeto dentro del entorno virtual. El tratamiento fue de 2 sesiones ( 2 minutos cada una). El instrumento de evaluación empleado fue el $S A D S$ (ver tabla 2).

En los estudios 8 (Klinger et al., 2005) y 23 (Wallach, Safir \& Bar-Zvi, 2009) -véase tabla 1-, cada paciente del grupo de RV asistió a 12 sesiones de terapia virtual que consistieron en presenciar diversos mundos virtuales generadores de ansiedad social. La jerarquía de la Exp podía ser modulada por el terapeuta. En el primer estudio mencionado los instrumentos de evaluación fueron el BDI-13, CGI, $H A D, L S A S, R A S, S C I A$, SISST y SIS. En el estudio 23 los instrumentos han sido el FNE, LSAS y SSPS (ver tabla 2). En el estudio de Klinger et al. (2005), 18 pacientes $(N=18)$ fueron seleccionados para el grupo tratado y otros 18 como grupo control con un TCC convencional. La duración de la visualización dentro de la RV debía ser inferior a los 20 minutos. En el estudio de Wallach et al. (2010), 28 sujetos $(N=28)$ conformaron el grupo sometido a RV y 30 sujetos conformaron un segundo grupo experimental recibiendo 
sólo TCC convencional. También incluía un grupo control en lista de espera $(\mathrm{N}=30)$.

En el estudio 11 -ver tabla 1- de Garau, Slater, Pertaub \& Razzaque (2005) se empleó un diseño inter-grupos de cuatro factores que dependían del grado de respuesta de los avatares. Dichas condiciones fueron las siguientes: Condición 1 (Estática): Todos los avatares se encontraban estáticos, quietos en posición de lectura $(N=11)$; Condición 2 (Movimiento): Los avatares estaban animados, parecían personas normales en una sala de lectura $(N=10)$; Condición 3 (Respuesta): Las conductas de la condición 2 eran suplementadas por respuesta del avatar al participante. Cuando el participante se acercaba al avatar, éste podría responder cambiando de postura y dirigiendo una mirada $(N=10)$; Condición 4 (Hablando): Se mantenía la condición 3 pero ahora el avatar podría hablarle brevemente al participante $(n=10)$. Las variables de respuesta consideradas para el estudio fueron: la copresencia, la conducta del participante y la percepción de conciencia de los avatares. Como variables objetivas se midieron la actividad electrodérmica y la tasa cardiaca. El POMS y SADS fueron los instrumentos de evaluación empleados, como se refleja en las tablas 1 y 2 adjuntas. El tamaño muestral total del grupo experimental ha sido de 41 sujetos $(N=41)$.

En el estudio 12 -tabla 1- (Slater et al., 2006), el participante era inmerso en una escena virtual que consistía en un bar con un barman y dos parejas virtuales. Estos avatares (2 mujeres y 2 hombres) daban la impresión de tener consciencia de la presencia del sujeto y frecuentemente dirigían su mirada hacia él. La muestra estaba formada por 20 sujetos $(N=20)$ y estos disponían de sensores adecuados para captar las variables fisiológicas que pretendían ser medidas. La sesión constaba de 3 fases: la primera era el establecimiento de la línea base -2' aprox.-, la segunda de entrenamiento y finalmente, el experimento -estas últimas con una duración de 15' aprox.-. El $S P Q$ (ver tabla 2) fue el instrumento de evaluación empleado.

En el estudio 13 -ver tabla 1-(Grillon, Riquier, Herbelin \& Thalmann, 2006), el experimento constaba de dos fases: La fase A - de no intervención-donde se estableció la curva de evolución de los síntomas diana a través de 3 sesiones de evaluación y, la fase B -fase de intervención- donde se exponía a los sujetos a situaciones provocadoras de ansiedad a través de la inmersión con $H M D$. El grupo experimental constaba de 8 sujetos $(N=8)$ que fueron expuestos durante 8 sesiones a escenas dentro de una oficina, observando a un avatar masculino y otro femenino; otra, una oficina con 5 avatares que daban la impresión de estar trabajando y luego un auditorio, observando primero a un avatar masculino y luego a otro femenino y luego a 20 avatares sentados en el fondo del recinto. Finalmente se mostraban imágenes avatares dentro de una cafetería y un bar. Los instrumentos de evaluación empleados fueron el $B D I, F Q, L S A S$ y SISST (ver tabla 2).
En el estudio 22 reflejado en la tabla 1 adjunta (Brinkman, Inan \& Van-Der-Mast, 2009), dentro de una habitación creada virtualmente con varios avatares sentados viendo una película en una pantalla, el experimento consistió en realizar dos rutas animadas siguiendo un orden establecido. Dicho orden tenía que ver con el color y la colocación de las sillas dentro del recinto, lo que elicitaba distintos niveles de ansiedad en el paciente que debía introducirse en la escena virtual. El número de participantes fue de 22 sujetos, si bien tan sólo 3 fueron diagnosticados de TAS $(N=3)$ y sólo se llevó a cabo una sesión de exposición a RV. Los instrumentos de evaluación utilizados fueron el $I P Q$ y el $L S A S$ (ver tabla 2).

Finalmente, en el estudio 24 -tabla 1- (Dumoulin, Bouchard, Robillard, Guitard \& Klinger, 2010), los participantes $(\mathrm{N}=45)$ fueron asignados aleatoriamente a tres condiciones: a) TCC tradicional con Exp en vivo (N=17); b) TCC combinado con RV ( $N=14)$; c) Lista de Espera $(\mathrm{N}=15)$, tratado posteriormente con un tratamiento combinado a base de Exp in vivo e in virtuo. Este estudio aún no se encuentra publicado.

- Entrevistas de trabajo: Es una situación que genera una elevada intensidad en las respuestas de ansiedad. El contacto es directo, provocando reacciones fisiológicas perturbadoras del comportamiento del paciente. Así, el entorno virtual con avatares dentro de una oficina, puede simular una situación social donde el sujeto debe actuar.

Los estudios 6 y 14 (véase tabla 1) consistieron en diseños factoriales $2 \times 2$. En el estudio 6 (Garau, Slater, Vinayagamoorthy, Brogni, Steed \& Sasse, 2003) los sujetos se asignaron aleatoriamente y las variables de respuesta se midieron para cuatro situaciones de interacción dentro del escenario virtual: cara a cara, participación, copresencia y evaluación social. El participante disponía de un HMD. En el estudio 14 (Pan \& Slater, 2007), el primer factor fue ser paciente sin TAS y el segundo con TAS. El tercer y cuarto factores fueron si eran o no observados por otras personas en el mundo virtual respectivamente. Los instrumentos de medida utilizados fueron el SADS (estudio 6) y SPAI (estudio 14).

El estudio 19 -tabla 1- (Kang, Gratch, Wang \& Watt, 2008 citado en Müeller \& Parsons) fue diseñado con cuatro condiciones: $1^{\circ}$ Cara a cara: El participante hablaba a un humano que lo escuchaba; $2^{\circ}$ Condición mediada: El participante interactuaba con un avatar que movía la cabeza y reflejaba posturas humanas; $3^{\circ}$ Condición receptiva: El sujeto interactuaba con un avatar que mostraba comportamientos de acuerdo a su voz y movimientos de cabeza; $4^{\circ}$ Condición no contingente: El sujeto interactuaba con un avatar que no modificaba su actitud. El tamaño muestral fue de 131 sujetos $(N=131)$ y los instrumentos de evaluación el $E S, S R S, S P S$ y $T L S$ (ver tabla 2).

En el estudio 20 -tabla 1- (Mühlberger, Wieser \& Pauli, 2008), el experimento consistió en ver la atención 
inicial de los participantes ante dos situaciones: la primera era dirigir su mirada a un objeto y un avatar y la segunda, hacia dos avatares que se le presentaban en una situación social ansiosa. El grupo tratado tenía un tamaño muestral de $N=12$, mientras que el grupo control (lista de espera) era de $\mathrm{N}=14$. Los instrumentos utilizados fueron el PANAS, SPAI, STAI-S y STAI-T (véase tabla 2).

- Entorno a través de Internet/Ordenador: Enfocado al campo de la "Telepsicología", Internet se muestra como una herramienta potencialmente válida para integrarse como un elemento más o como alternativa a la Exp en vivo en el tratamiento del TAS; esta última mediante el empleo de terapias "virtuales" que pueden encontrarse en la red o visualizarse en el ordenador.

En el estudio 15 de la tabla 1 (Anderson, Zimand, Schmertz \& Ferrer, 2007), se realizó un tratamiento de 8 sesiones con un tamaño muestral de 10 sujetos $(N=10)$, combinando TCC y RV. Los pacientes recibieron 4 sesiones de TCC y 4 sesiones de RV frente a una audiencia virtual presentada en el monitor de un ordenador. Se emplearon diversos instrumentos de evaluación tales como el $B D I$, FNE, PRCA, PRCS, SSPS-P, SSPS-N, STAI-S y STAI-T (ver tabla 2).

En el estudio 16 (Botella et al., 2007) -según tabla 1-, el tratamiento consistió de 3 componentes: Educativo, TCC y RV. Los escenarios "Talk to me" estaban compuestos por vídeos de audiencias reales en situaciones de hablar en público (un aula, un examen oral, una reunión de trabajo y un grupo de amigos). El tamaño muestral fue $N=12$, utilizándose el ADIS-IV-L, FPSQ, PSSEQ y SUDS (ver tabla 2) como instrumentos de evaluación. El promedio de sesiones fue de 7,58 según el estudio.

El estudio 17 de la tabla 1 (Carlbring, Gunnardsdóttir, Hedensjö, Andersson, Ekselius \& Furmark, 2007) consistió en módulos cognitivo-conductuales a través de Internet con una duración de 9 semanas. Cada módulo incluía información, ejercicios y actividades interactivas. Los participantes $(N=29)$ podían explicar con sus propias palabras lo más importante de cada sección y podía hacerse una llamada telefónica semanal al terapeuta. El propósito era ofrecer un feedback positivo y responder las cuestiones planteadas por el participante. Los instrumentos empleados fueron el $L S A S, B A I, M A D R S, S C I D$, SIAS, SPS y SPSQ (ver tabla 2). El grupo control (lista de espera) tenía un tamaño $\mathrm{N}=28$.

El estudio 18 (Tillfords et al., 2008) -según tabla 1contó con 2 grupos, ambos con TCC a través de Internet y uno de ellos, además, con Exp en vivo. El tamaño muestral fue de 65 sujetos $(N=65)$ y los instrumentos de evaluación del nivel de ansiedad han sido $L S A S, S P S, S I A S$ y $S P S Q$ (tabla 2).

En el estudio 21 -ver tabla 1- (Berger, Holh \& Caspar, 2009), la intervención consistió en un programa basado en Internet de 10 semanas de duración, que incluía una guía interactiva de auto-ayuda, un módulo para establecer contacto regular con un terapeuta, un sistema continuo de feedback y monitorización de las respuestas del paciente así como un grupo de colaboración online, ofreciendo la oportunidad de compartir experiencias entre participantes. El grupo experimental contó con 31 sujetos $(N=31)$ y el grupo control (lista de espera) con $21(\mathrm{~N}=21)$. Los instrumentos de evaluación fueron el BDI, LSAS, SIAS, SPS y $S C L-90-R$ (según tabla 2).

\section{Discusión}

\section{Realizando una revisión de cada uno de los estudios, puede llegarse a las siguientes conclusiones:}

Estudios relacionados con el miedo a hablar en público: Se han contabilizado 6 estudios según lo reflejado en la tabla 1 (estudios 1, 2, 3, 7, 9 y 10). El estudio 1 indica que la RV es eficaz en la reducción de la ansiedad según los datos aportados por las medidas de auto-informes realizadas. Su principal limitación es su bajo tamaño muestral. Los resultados encontrados para el estudio 2 apoyan el uso de la RV para el tratamiento de personas que sufren TAS, según resultados arrojados en el PRCS (véase tabla 2). En el estudio 3, los sujetos respondieron a las audiencias-seminario virtuales tal como lo harían con las audiencias reales. Además, indicaron que se sentían mejor frente a un grupo de espectadores que les retroalimentaba positivamente, sintiendo un considerable malestar al hablar ante una audiencia de aspecto desagradable. Con las audiencias virtuales y el escenario de la audiencia negativa en particular, la co-presencia fue suficiente para elicitar respuestas afectivas intensas en los sujetos que debían hablar ante los avatares. La medida de la confianza para hablar en público obtenida después de la charla, permitió evaluar la intensidad de las respuestas de ansiedad al hablar en público, la cual fue particularmente intensa ante la audiencia de avatares que retroalimentaba negativamente la actuación de los participantes.

En el estudio 7 se ha demostrado que una respuesta fuerte de presencia se puede obtener con una fidelidad relativamente baja de un entorno virtual que representa un encuentro social (en este caso, una persona que da una charla). Los hablantes con TAS mostraron diferencias en la ansiedad de varias formas (los que hablaron a la audiencia informaron de la ansiedad basándose en aspectos del cuestionario que fueron significativamente mayores que los que hablaron a la sala vacía, siendo la valoración subjetiva de su respuesta somática casi el doble). Para el estudio 9, los resultados obtenidos en el PRCS (véase tabla 2) permiten concluir que la RV dentro de los TCC ha sido efectiva para el tratamiento del TAS. En el estudio 10 se han considerado dos grupos: uno con marcada fobia social y otro con mediana ansiedad social. Para ambos grupos se 
ha podido constatar que el tratamiento empleando RV ha sido altamente eficaz.

Estudios relacionados con entornos públicos virtuales: Se han extraído 9 estudios según tabla 1 (estudios 4, 5, 8, $11,12,13,22,23$ y 24). En el estudio 4 se contó con un tamaño muestral muy pequeño $(\mathrm{N}=4)$, lo que no permite concluir estadísticamente que el empleo de RV en el tratamiento del TAS sea eficaz. En el estudio 5 han resultado significativas las respuestas fisiológicas subjetivas encontradas en los sujetos tras la aplicación del tratamiento de RV. En el estudio 8 se reportan diferencias significativas entre el pretest y el postest en el LSAS (ver tabla 2) para los valores de ansiedad y evitación, lo que permite concluir la efectividad de RV para el tratamiento del TAS. El objetivo del experimento desarrollado en el estudio 11 era aislar conductas no-verbales de interacción verbal para comprender como la co-presencia podía estar afectada por pequeños incrementos de respuesta en humanos virtuales. Así, en la condición 3 del experimento (cuando los avatares interactuaban con el participante) aumentaba significativamente el sentido de contacto personal con los mismos, si bien este efecto se ve disminuido en pacientes habituados al uso de los ordenadores. En el estudio 12, los resultados se han tomado de respuestas fisiológicas medidas en los sujetos ante la Exp en RV, tales como la tasa cardiaca. En la investigación realizada no se obtuvieron valores estadísticamente significativos, luego considerar solamente la tasa cardiaca no es un predictor fiable para la eficacia de la RV en pacientes con TAS. En el estudio 13, ha podido comprobarse que los valores de ansiedad reportados en los diferentes instrumentos de evaluación psicológica aplicados, disminuyeron tras la aplicación del tratamiento en RV.

Con el fin de comprobar si el entorno prototípico virtual recrea una situación de la vida real con éxito y es capaz de provocar la misma respuesta humana, en el estudio 22 se investigaron dos casos de pacientes fóbicos -un tamaño muestral muy pequeño para llegar a conclusiones válidas-. En el estudio en cuestión, tan sólo se dispone de 3 sujetos con fobia social, lo que tampoco permitió obtener resultados estadísticamente significativos. Para el estudio 23 puede llegarse a la conclusión de que el componente de RV dentro del TTC para pacientes con TAS resulta beneficioso para la terapia. Según los autores del trabajo, el componente de "Exp" dentro de un TCC, tanto en vivo como en imaginación, puede presentar serias dificultades tanto para el paciente como para el terapeuta, por lo que el empleo de la RV ofrece una alternativa atractiva para paliar estas posibles dificultades. Finalmente, en el estudio 24 (aún no publicado) el autor ha enviado sus primeros resultados como adelanto al trabajo final que culminará a finales del año 2011. No obstante, los autores parecen sugerir que tras los primeros resultados, la $\mathrm{RV}$ es una herramienta efectiva en el tratamiento del TAS y que combinando Exp tradicional con RV pueden obtenerse avances terapéuticos muy significativos.

Estudios relacionados con simulación de entrevistas de trabajo: Se han contabilizado 4 estudios según lo reflejado en la tabla 1 (estudios 6, 14, 19 y 20). En el estudio 6 se ha tomado la animación de la mirada como una instancia específica de la conducta avatar. No puede afirmarse, por supuesto, que los resultados puedan generalizarse a otros aspectos de la conducta avatar, pero con la mirada del avatar se ha pretendido estudiar aspectos significativos de su conducta que puedan provocar ansiedad en el sujeto experimental. Los resultados encontrados no han sido significativos por lo que la investigación deberá ir más allá en este tipo de variables. En el estudio 14, el grupo ansioso presentaba una respuesta de ansiedad mayor que los integrantes del grupo seguro y dentro del grupo seguro se apreció que la ansiedad era mayor cuando la persona era observada. Por tanto, la sensación de presencia juega un papel fundamental en provocar reacciones ansiosas en los pacientes y su modulación a través de técnicas de RV puede resultar beneficiosa para pacientes que estén en tratamiento de TAS. En el estudio 19 se ha explorado si la contingencia en el feedback no-verbal mostrada por los avatares, puede estar relacionada entre la ansiedad social de los participantes y sus sentimientos manifestados en relación a diversos patrones de interacción. Los resultados muestran que en la condición no-contingente, tanto el auto-rendimiento como la reducción de la vergüenza sufren mejoras significativas. De igual forma, la confianza se reduce en la condición cara a cara. Esto indica que las personas con mayor ansiedad social confían menos en patrones de interacción con humanos reales, dando apoyo a la opinión de que los humanos virtuales (avatares) y los entornos virtuales, pueden facilitar la interacción entre personas con TAS y el consiguiente beneficio terapéutico. Para el último estudio de este grupo (20), se demostró que la atención inicial y sostenida difieren. $\mathrm{La}$ atención sostenida, sin embargo, fue influenciada por la inducción experimental de miedo social, de tal manera que los participantes que se atrevieron a dar una charla pública desplegaron su atención más hacia caras felices que a caras enojadas. Por lo tanto, las personas con ansiedad parecen evitar la atención sostenida a los rostros enojados.

Estudios relacionados con Internet/Ordenador o PC: Se han extraído 5 estudios, según la tabla 1 marcados con los números $15,16,17,18$ y 21 . En el estudio 15 , los resultados de este ensayo clínico abierto sugieren que los participantes encontraron útil y práctico el programa de auto-ayuda informatizado para combatir el TAS. En el estudio 16, los autores ofrecen evidencia empírica preliminar sobre la utilidad de "Talk to me", un programa de auto-ayuda telepsicológica para el tratamiento del temor de hablar en público. Consistente con el objetivo principal de la investigación, los datos reflejan un cambio significativo entre el pre y el 
post-tratamiento en las medidas directamente relatadas para miedo a hablar en público. Los resultados arrojados en el estudio 17 sugieren que el grupo tratado experimentó una reducción significativa de las medidas de ansiedad general y social, evitación y depresión. La adherencia al tratamiento fue alta (93\%) según reportan los autores. Este estudio proporciona pruebas que apoyan el uso de un tratamiento basado en Internet complementado por teléfono con llamadas semanales cortas para el tratamiento eficaz del TAS. El estudio 18 examinó la eficacia de un programa de auto-ayuda cognitivo-conductual administrado a través de Internet, a una muestra de estudiantes con fobia social en una Universidad de Suecia, y específicamente comprobó la hipótesis de que un programa podría ser más eficaz, cuando combinaba sesiones de Exp en vivo grupales en relación a un programa con tratamiento exclusivamente a través de Internet. Para concluir, los resultados del estudio 21 sostienen la noción de que los TCC junto con el empleo de Internet y un contacto mínimo con el terapeuta, pueden ser una prometedora herramienta terapéutica para el tratamiento del TAS (el tratamiento basado en Internet se mostró estadísticamente significativo en todas las medidas de ansiedad social reportadas en el LSAS, SPS, y SIAS -ver tabla 2-).

Para concluir, del análisis de los estudios referenciados se desprende que, en general, la inclusión de la RV y/o Internet dentro de los tratamientos psicológicos, se muestra muy prometedora en el tratamiento del TAS, si bien una de las limitaciones más relevantes de estos estudios ha sido el reducido tamaño de la muestra utilizada.

\section{Referencias}

(con * forman parte de la revisión)

American Psychiatric Association (2000). Diagnostic and Statistical Manual of Mental Disorders (DSM-IV-TR). Washington, D.C.: APA.

Amerigen, M., Mancini, C. \& Farvolden, P. (2003). The impact of anxiety disorders on educational achievement. Journal of Anxiety Disorders, 17, 561-571.

*Anderson, P.L., Zimand, E., Hodges, L.F. \& Rothbaum, B. O. (2005). Cognitive behavioral therapy for public-speaking anxiety using virtual reality for exposure. Depression and Anxiety 22, 156-158.

*Anderson, P., Zimand, E., Schmertz, S. \& Ferrer, M. (2007). Usability and utility of a computerized cognitive-behavioral self-help program for public speaking anxiety. Cognitive and Behavioral Practice, 14, 198-207.

Bados, A. (2009). Fobia social. Facultad de Psicología. Departament de Personalitat, Avaluaciò i Tractaments Psicològics. Universitad de Barcelona. Extraído en julio, 7 de 2009, disponible en http://diposit. ub.edu/dspace/bitstream/2445/6321/1/Fobia\%20social.pdf

Beidel, D. C. \& Turner, S. M. (2005). Childhood anxiety disorders. A guide to research and treatment. New York: Routledge.

*Berger, T., Holhl, E. \& Caspar F., (2009). Internet-based treatment for social phobia: A randomized controlled trial. Journal of Clinical Psychology, 65, 1021-1035.

*Botella, C., Guillén, V., García-Palacios, A., Gallego, M.J., Baños, R.M. \& Alcañiz, M. (2007). Telepsychology and self-help: The treatment of fear of public speaking. Cognitive and Behavioral Practice, 14, 46-57.

*Brinkman, W.P., Inan, F. \& Van-der-Mast, Charles A.P.G. (2009, April) A virtual environment to create social situations: First step to a virtual reality exposure therapy system for social phobia. Euromedia Conference, pp. 103-107, Ostend, Belgium.

*Carlbring, P., Gunnarsdöttir, M., Hedensjö, L., Andersson G., Ekselius L. \& Furmark, T. (2007). Treatment of social phobia: Randomised trial of internet-delivered cognitive-behavioural therapy with telephone support. British Journal of Psychiatry, 190,123-128.

*Dumoulin, S., Bouchard, S., Robillard, G., Guitard, T. \& Klinger, E. (2010). Early results from a randomized trial on efficacy of virtual reality to treat social anxiety. Canadá: University of Quebec.

Eng, W., Coles, M. E., Heimberg, R. G. \& Safren, A. S. (2001). Quality of life following cognitive behavioral treatment for social anxiety disorder: Preliminary findings, Depression and Anxiety, 13, 192-193

Federoff, I. C. \& Taylor, S. (2001). Psychological and pharmacological treatments of social phobia: A meta-analysis. Journal of Clinical Psychopharmacology, 21, 311-24.

Feske, U. \& Chambless, D. L. (1995). Cognitive behavioral versus exposure only treatment for social phobia: A meta-analysis. Behavior Therapy, 26, 695-720.

Furmark, T., Tillfors, M., Marteinsdottir, I., Fischer, H., Pissiota, A., et al. (2002). Common changes in cerebral blood flow in patients with social phobia treated with citalopram or cognitive-behavioral therapy. Archives of General Psychiatry, 59, 425-433.

*Garau, M., Slater, M., Pertaud, D.P. \& Razzaque, S. (2005). The Responses of people to virtual humans in an immersive virtual environment. Presence, Teleoperators and Virtual Environments, 14, 104-116.

* Garau, M., Slater, M., Vinayagamoorthy, V., Brogni, A., Steed, A. \& Sasse, A.M. (2003). The impact of avatar realism and eye gaze control on perceived quality of communication in a shared immersive virtual environment. Proceedings of Conference on Human Factors in Computing Systems (CHI2003), Lauderdale, Florida.

Gould, R. A., Buckminster, S., Pollack, M. H., Otto, M. W. \& Yap, L. (1997). Cognitive-behavioral and pharmacological treatment for social phobia: A meta-analysis. Clinical Psychology: Science and Practice, 4, 291-306

Greenberg, P. E., Sisitsky, T., Kessler, R. C., Finkelstein, S. N., Berndt, E. R., Davidson, J. R. T., Ballenger, J. C. \& Fyer, A. J. (1999). The economic burden of anxiety disorders in the 1990s. Journal of Clinical Psychiatry, 60, 427-435.

*Grillon, H., Riquier, F., Herbelin, B. \& Thalmann, D. (2006). Virtual reality as therapeutic tool in the confines of social anxiety disorder treatment. International journal on disability and human development, 5, 243-250.

*Grillon, H., Riquier, F., Herbelin, B. \& Thalmann D. (2006, September). Use of virtual reality as therapeutic tool for behavioural exposure in the ambit of social anxiety disorder treatment. The 6th International Conference Series on Disability, Virtual Reality and Associated Technologies, pp. 105-112, Esbjerg, Dernmark. (disponible en http://infoscience.epfl. ch/record/99738/files/Grillon_and_al_ICDVRAT_06.pdf)

Hambrick, J. P., Turk, L. C., Heimberg, R. G., Schneier, F. R. \& Liebowitz, M. R. (2003). The experience of disability and quality of life in social anxiety disorder. Depression and Anxiety, 18, 46-50

*Harris, S.R., Kemmerling, R. L. \& North, M.M. (2002). Brief virtual reality therapy for public speaking anxiety. Cyberpsychology \& Behavior, 5, 543-550.

*Herbelin, B. (2005). Virtual reality exposure therapy for social phobia. Thesis $N^{\circ} 3351$, VRLab, Faculté informatique et communications, Institut des systèmes informatiques et mutlimédias, EPFL, Switzerland.

*James, L. K., Lin, C-Y., Steed, A., Swapp, D. \& Slater, M. (2003). Social anxiety in virtual environments: Results of a pilot study. Cyberpsychology \& Behavior, 6, 237-243.

*Kang, S.H., Gratch, J., Wang, N. \& Watt, J.H. (2008). Does the Contingency of Agents? Nonverbal Feedback Affect Users? Social Anxiety? En L. Padgham, D. Parkes, J. Müller \& S. Parsons (Eds.), Proceedings of 7th International Conference on Autonomous Agents and Multiagent Systems (AAMAS 2008) (pp. 120-127). Estoril, Portugal: IFAAMS

Katon, W. \& Geyman J. P. (2007). Anxiety disorders. En R. E. Rakel y D. Rakel (Eds.),Textbook of family medicine (7th ed.) (pp. 321-375). Philadelphia, Pa (USA): Saunders Elsevier. 
Kessler, R. C. (2003). The impairments caused by social phobia in the general population: implications for intervention. Acta Psychiatrica Scandinavica, 108, (Suppl. 417), 19-27.

Kessler, R. C., Berglund, P., Demler, O., Jin, R. \& Walters, E. E. (2005). Lifetime prevalence and age-of-onset distributions of DSM-IV disorders in the national comorbidity survey replication. Archives of General Psychiatry, 62, 593-602.

Kessler, R. C., Chiu, W. T., Demler, O. \& Walters, E. (2005). Prevalence, severity, and comorbidity of 12-month DSM-IV disordersin the national comorbidity survey replication. Archives of General Psychiatry, 62, 617-627.

*Klinger, E., Bouchard, S., Légeron, P., Roy, S., Lauer, F., Chemin, I. \& Nugues, P. (2005). virtual reality therapy versus cognitive behavior therapy for social phobia: A preliminary controlled study. CyberPsychology \& Behavior, 8, 76-88.

Krijn, M., Emmelkamp, P.M.G., Olafsson, R.P. \& Biemond, R. (2004). Virtual reality exposure therapy of anxiety disorders: A review. Clinical Psychology review, 24, 259-281.

Lee, J.M., Ku, J.H., Jang, D.P., Kim, D.H., Choi, Y.H., Kim, I.Y. \& Kim, S.I. (2002). Virtual reality system for treatment of the fear of public speaking using image-based rendering and moving pictures. Cyberpsychology \& behavior, 5, 191-196.

*Mühlberger, A., Wieser, M. \& Pauli, P. (2008). Visual attention during virtual social situations depends on social anxiety. Cyberpsychology and Behavior, 11, 425-430.

*North, M.M., North, S.M. \& Coble, J.R. (1998). Virtual reality therapy: An effective treatment for the fear of public speaking. International Journal of Virtual Reality, 3, $2-7$.

Olasov, B. \& Hodges, L. (1999) The use of virtual reality exposure in the treatment of anxiety disorders. Behavior Modification, 23, 507-525.

Olivares, J. (2005). El niño con miedo a hablar (5 $5^{\mathrm{a}}$ Ed.). Madrid: Pirámide.

Olivares, J. (2009). Evaluación y tratamiento de la fobia social en la población infanto-juvenil de habla española y portuguesa. Anuario de Psicología, 40, 7-21.

Olivares, J., Rosa, A. I. \& Piqueras, J. (2005). Detección temprana y tratamiento de adolescentes con fobia social. Psicothema, 17, 1-8.

Olivares-Olivares, P. J., Rosa-Alcázar, A. I. \& Olivares, J. (2007). Validez social de la intervención en adolescentes con fobia social: Padres frente a profesores. Terapia Psicológica, 25, 63-71

Rosa-Alcázar, A. I., Olivares, J. \& Olivares-Olivares, P. J. (2007). Papel de la planificación de la interacción en el tratamiento psicológico de adolescentes con fobia social generalizada. Terapia Psicológica ,25, 205-212.

* Pan, X. \& Slater, M. (2007, mes). A Preliminary study of shymales interacting with a virtual female. The 10th Annual International Workshop on Presence, pp. 101-108, Barcelona, Spain.

Parsons, T.D. \& Rizzo, A.A. (2008). Affective outcomes of virtual reality exposure therapy for anxiety and specific phobias: A meta-analysis. Journal of Behavior Therapy and Experimental Psychiatry, 39, 250-261.
Pine, D. S., Cohen, P., Gurley, D., Brook, J. \& Ma, Y. (1998). The risk for earlyadulthood anxiety and depressive disorders in adolescents with anxiety and depressive disorders. Archives of General Psychiatry, 55, 56-64.

*Pertaub, D.P, Slater, M. \& Barker, C. (2001). An experiment on fear of public speaking in virtual reality. Studies in health technology and informatics, 81, 372-378.

*Pertaub, D.P., Slater, M. \& Barker, C. (2002). An experiment on public speaking anxiety in response to three different types of virtual audience. Presence, Teleoperators and Virtual Environments, 11, 68-78.

Rao, P. A., Beidel, D. C., Turner, S. M., Ammerman, R. T., Crosby, L. E. \& Sallee, F. R. (2007). Social anxiety disorder in childhood and adolescence: Descriptive psychopathology. Behaviour Research and Therapy, 45, 1181-1191.

Rapee, R. M. \& Spence, S. H. (2004). The etiology of social phobia: Empirical evidence and an initial model. Clinical Psychology Review, 24, 737-767.

*Roy, S., Klinger, E., Legeron, P., Lauer, F., Chemin, I. \& Nugues, P. (2003). Definition of a VR-based protocol to treat social phobia. $C y$ berpsychology \& Behavior, 6, 411-420.

Ruscio, A. M., Brown, T. A., Chiu, W. T., Sareen, J., Stein, M. B. \& Kessler, R. C. (2008). Social fears and social phobia in the USA: Results from the national comorbidity survey replication. Psychological Medicine, $38,15-28$.

Safren, A. S.. Heimberg, R. G., Brown, E. J. \& Holle, C. (1997). Quality of life in social phobia. Depression and Anxiety, 4, 126-133

*Slater, M., Pertaud, D.P., Barker, C. \& Clark, D. (2004, September). An experimental study on fear of public speaking using a virtual environment. 3rd International Workshop on Virtual Rehabilitation IWVR 2004, Lausanne, Switzerland.

* Slater, M., Guger, C., Edlinger, G., Leeb, R., Pfurtscheller, G., Antley, A., Garau, M., Brogni, A. \& Friedman, D.(2006). Analysis of physiological responses to a social situation in an immersive virtual environment. Teleoperators and Virtual Environments, 15, 553-569.

Taylor, S. (1996). Meta-analysis of cognitive-behavioral treatment for social phobia. Journal of Behavior Therapy and Experimental Psychiatry, 27, 1-9.

*Tillfords, M., Carlbring, P., Furmark, T., Lewenhaupt, S., Spak, M., Eriksson, M., Westling, B. \& Andersson, G. (2008). Depression and Anxiety, 25, 708-717.

*Wallach, H.S., Safir M.P. \& Bar-Zvi, M. (2009). Virtual reality cognitive behavior therapy for public speaking anxiety: A randomized clinical trial. Behavior Modification, 33, 314-338.

Wittchen, H.U., Feutsch, M., Sonntag, H., Muller, N. \& Liebowitz, M. (2000). Disability and quality of life in pure and comorbid social phobia: Findings from a controlled study. European Psychiatry, 15, 46-58. 